\title{
Isolation and Diagnosis of Fungi Associated With Citrus Fruits Taken From The Local Markets of The City of Mosul and Laboratory Control
}

\author{
Rafia K. Gergis \\ Nisreen I. Mohamed \\ University of Mosul / College of Education for Pure Sciences \\ Department of Life Sciences \\ rafiajarjes@yahoo.com \\ DOI: $\underline{10.33899 / \text { edusj. } 1970.163326}$ \\ nisreenmohammed@gmail.com \\ Received \\ $06 / 01 / 2018$ \\ Accepted \\ $30 / 07 / 2018$

\begin{abstract}
The present study involved the isolation and diagnosis of molds and yeasts which affecting the local and imported citrus fruits available in the local markets of the Mosul. These fruits include (grapefruit, oranges, lemons, sweet limes, oranges Japanese, sour, bitter orange and tangerine) .45 isolates were obtained from filamentous fungi and 26 isolates also were obtained from yeasts. The fungi were identified morphogically and microscopically based on Pitt and Hocking taxonomic index(2009) . The molds samples were distributed to the following species Penicillium, Aspergillus, Cladosporium, Mucor, Alternaria, Trichoderma, Fusarium, Rhizopus. The two genera Aspergillus and Penicillium were identified to the species level, because of their frequent ocurrence in the isolates, as well as the yeasts were diagnosed morphogically and microscopically, their growth have been studied on the differential medium. The system Vitek 2 Compact also has been used .Distinction was made between Basidiomycetes and Ascomycetes yeasts. yeasts samples were distributed to 5 genera.They are Crptococcus spp, Candida spp, Saccharomyces spp, Rhodotorula mucillaginosa, and Geotrichum candidum. The present study included the use of Candida guilliermondii (which has been isolated during the present study), yeast as biological control for the isolated yeast of the present study. The yeast showed high efficiently in inhibiting molds growth specially $P$. italicum and A. niger, the yeast inhibited their growth completely and it was $100 \%$.
\end{abstract}

Key word : fungi - yeasts - fruits 
عزل وتثخيص الفطريات المصاحبة لثمار الحمضيات المأخوذة من الاسواق المحلية لمدينة الموصل ومكافحتها مختبرياً

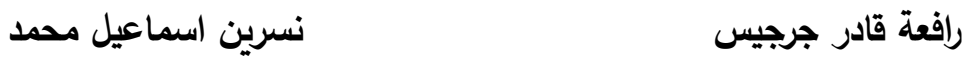 \\ جامعة الموصل ، كلية التربية للعلوم الصرفة ، قسم علوم الحياة
}

nisreenmohammed@gmail.com

rafiajarjes@yahoo.com

DOI: $\underline{10.33899 / \text { edusj.1970.163326 }}$

\begin{tabular}{|c|c|}
\hline القبول & الاستلام \\
\hline 2018 / 07 / 30 & $2018 / 01 / 06$ \\
\hline
\end{tabular}

\title{
الخلاصة
}

تتاولت الدراسـة الحالية عزل وتشخيص الأعفان والخمائر الملوثة لفاكهة الحمضيات المحلية والمستوردة والمتوفرة في الاسواق المحلية لمدينة الموصل والتي تتضمن(الكريب فروت او ليمون الجنة, البرتقال, الليمون

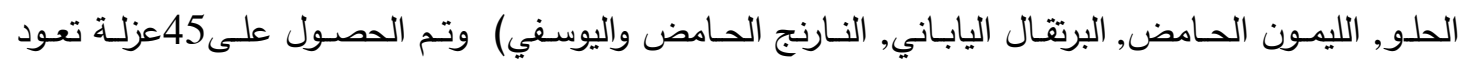
للاعفان(الفطريات الخيطية) و26 عزلة تعود للخمائر • وجرى تثخيص الفطريات المعزولة مظهرياً ومجهرياً

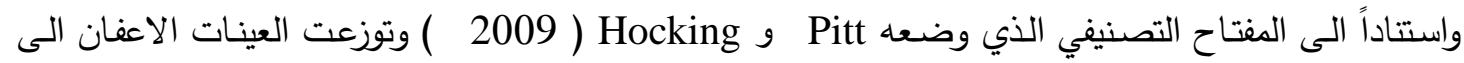

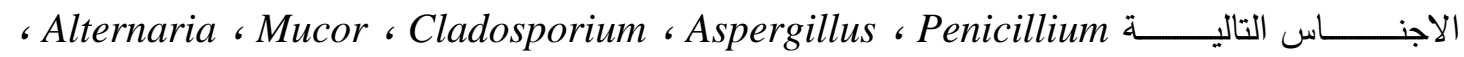
Rhizopus ، Fusarium ، Trichoderma مستوى النوع وذلك لكثرة تكرارهما بين الفطريات المعزولة .وكذلك تشخيص الخمائر المعزولة مظهرياً ومجهرياً ودراسـة نموهـا على الاوسـاط التقريقية وكذلك باستخدام نظام ل Vitek2Compact كما وجرى تميز الخمائر

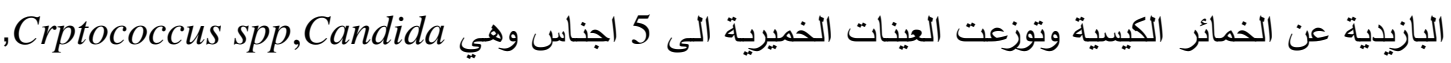
.Geotrichum candidum, Rhodotorula mucillaginosa spp, Saccharomyces ssp وتضمنت الدراسة الحالية ايضـاً استخدام الخميرة Candida guilliermondii (التي تم عزلها من بحثنا

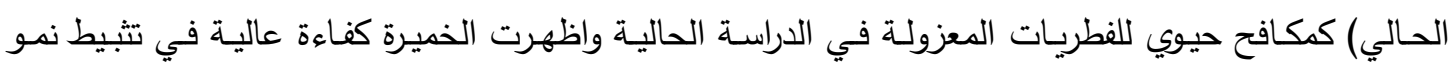

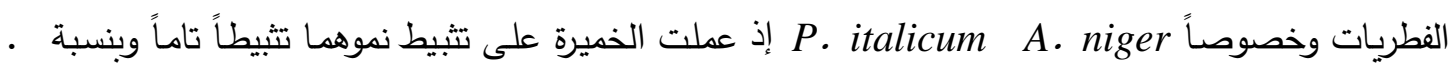




\section{Introduction المقدمة}

تعد الحمضيات من الفواكه ذات الاهمية الغذائية الكبيرة للانسان لما تحتويه من عناصر غذائية هامة اذ

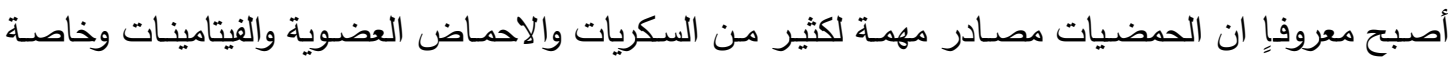

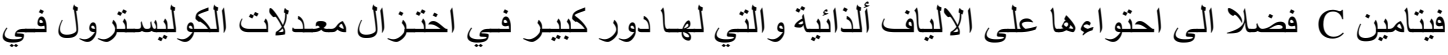
الجسم[1- 2 ] يعد الانتاج العالمي للحمضيات الاعلى من بين انواع الفاكهة الاخرئ. وفي جامعة فلوريدا

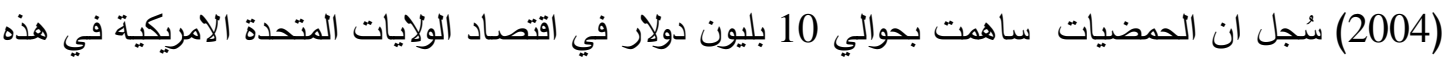

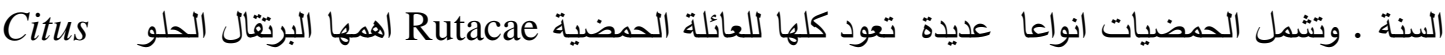
Citrus paradisi) والكريب فروت (ليمون الجنة Citrus aurantium والنارنج ( البرتقال المر sinensis

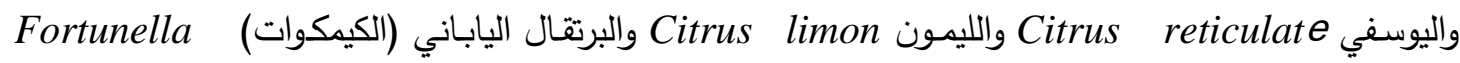
[3] margarite العصائر والمربيات , بالاضـافة الى حوالي 20\% من هذه الفاكهة يتم فقدانها كل عام بسبب التلف والاصسابة

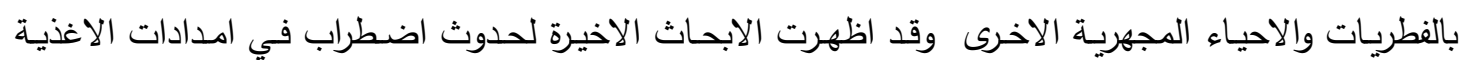

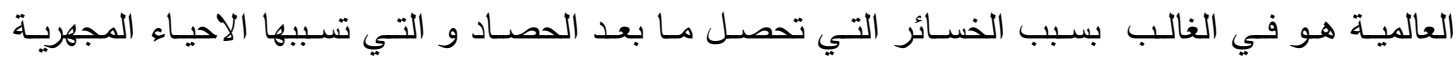

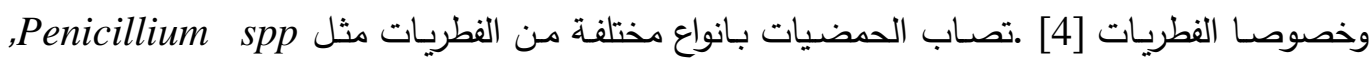

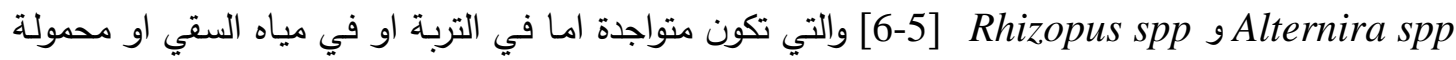
جوا وكذلك في معدات الحصاد والاشخاص العاملين بالحصاد [8-7], حيث تعمل هذه الفطريات عند نموها علئ الحمضيات الى اتلافها فضـلاٍ الىئ قدرة العديد منها وخاصـة الاذواع التابعة لكل من الجنسين Aspergillus, Alternirna

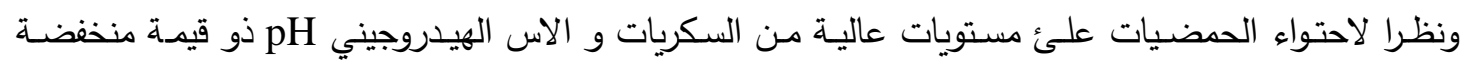

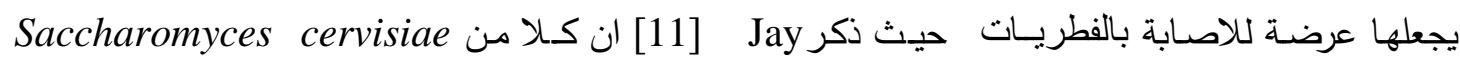
و Hansonula spp كانت مسوؤلة عن فساد الحمضيات (البرتقال, اليوسفي) في نيجيريا.كما سجلت اجناس

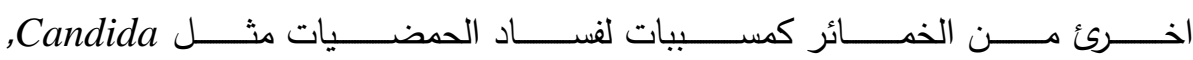

Trichosporan,Saccharomyces,Pichia,Kloeckera,Rhodoterula,Hansenula,Crptococcus [12] ونظرا لانفتاح الاسواق العراقية علئ الاسواق العالمية بدات تدخل كميات كبيرة من السلع الىئ البلد ومن

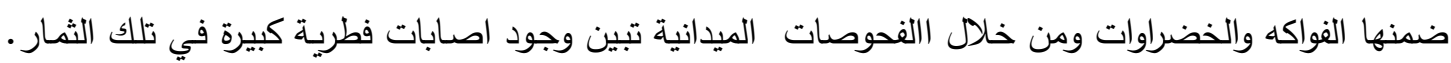

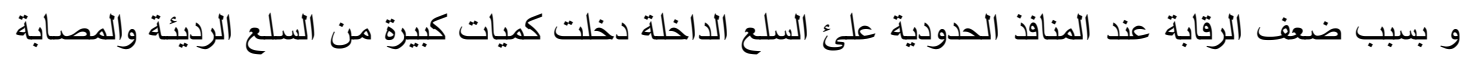

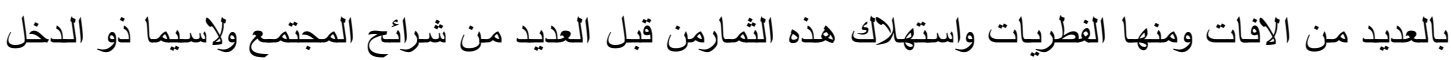

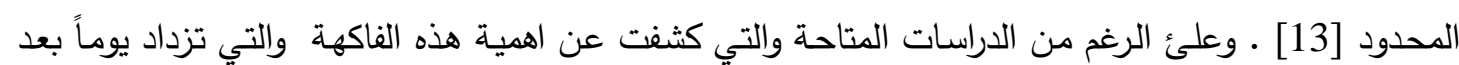

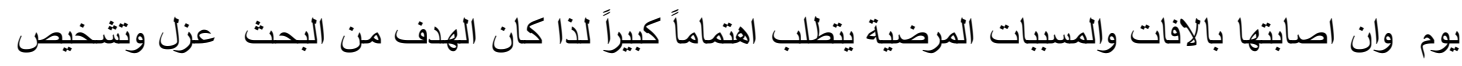

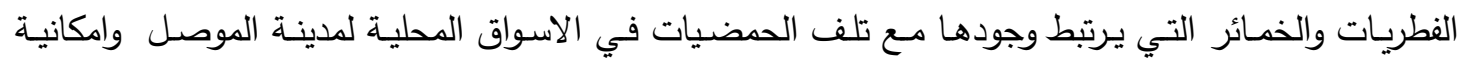
مكافحتها باستخدام عزلة من الخميرة المعزولة وهي Candida guilliermondii والتي تم الحصول عليها في 


\section{المواد وطرائق العمل}

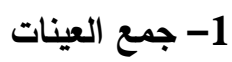

تم جمع عينات الفاكهة المصابة التي تبدوعليها اثار التعفن والنخر من الاسواق المحلية لمدينة الموصل ومن مناطق متفرقة (اسواق الجامعة واسواق النبي يونس والدركزلية وباب الطوب) اختيرت العينات عشوائيا

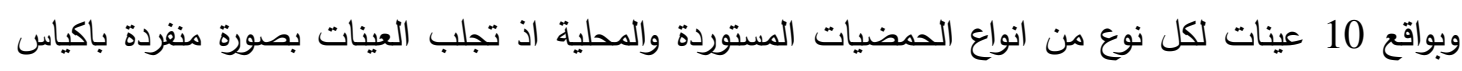
بولي اثيلين معقمة ونظيفة الى المختبر اذ كان وقت الجمع من كانون الاول الى اذار 2012.

2- الاوساط الزراعية المستخدمة في البحث

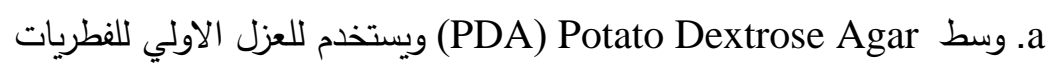
(CYA) Czapek Yeast extract Agar g. وسط وسط (MEA) Malt Extract Agar g.c

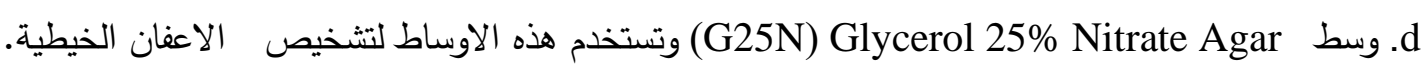
k. وسط و Malt - Meast extract Agar ) ويستخدم للعزل الاولي للخمائر (CA) CzapekAgar g.f (MAA) Malt Acetic Agar gسط.e

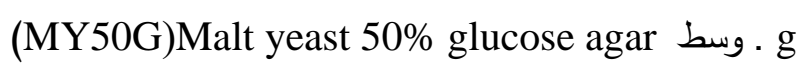

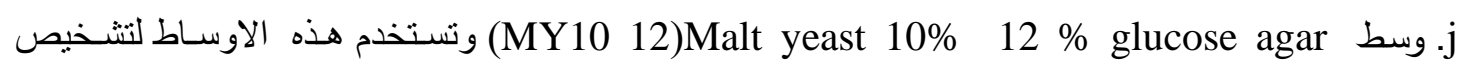
الخمائر[15]

3

يؤخذ الجزء المصاب من الثرة ويقسم الى قطع صغيرة (3-5)اجزاء وتوزع على ابعاد متساوية في طبق

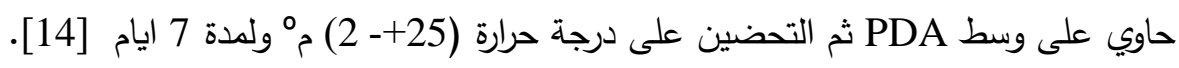

4- تنقية وتثخيص الفطريات جرى تتقية المستعمرات الفطريـة النامية على الوسط PDA ثم حفظت كل عينـة نقيـة على سطح مائل في

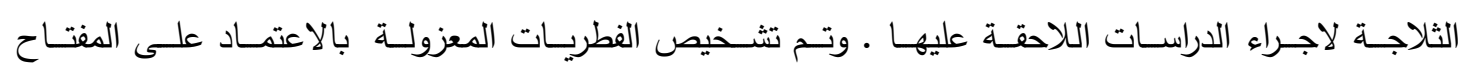
التصنيفي Pitt و Hocking [15] وذلك بملاحظة النمو على الاوساط الزرعية الثلاثة الأساسية للتثخيص وهي

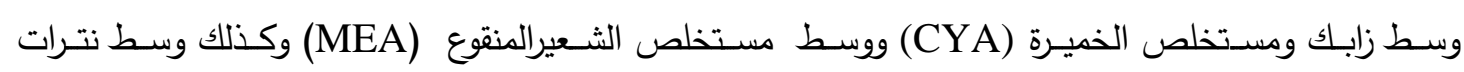

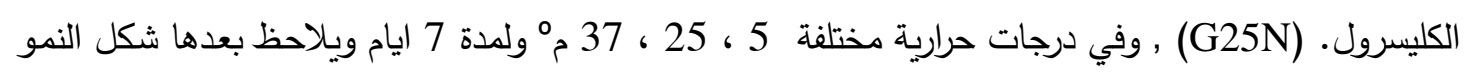
ولون المستعرة وقطرها.وكذلك الصفات المجهريـة شكل الخيوط الفطريـة, طريقة تفرعها ,وجود الحواجز رشكل الكونيدات ,عدد خلايا الكونيدات ,شكل الحامل الكونيدي,وجود الاجسام الحجرية وتراكيب اخرى.

5- 5ز عزل الخمائر

تم وضع الجزء المصاب من الفاكهة في بيكر زجاجي معقم ويضاف اليه 25 مليلتر من من الماء المقطر

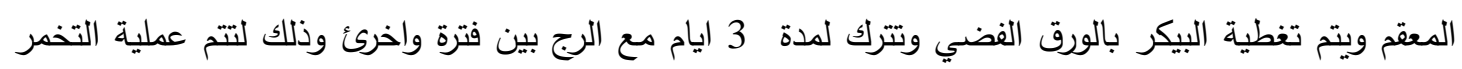


ويسهل عزل الخمائر وبعد 3 ايام سوف تظهر طبقة غشائية بيضاء علئ السطح وهذا دليل علئ حدوث التخمر و بعد ذلك يؤخذ 1 مليلتر من المعلق السابق ويضاف الى 9 مليلتر من الماء المقطر المعقم و يتم عمل بضعة

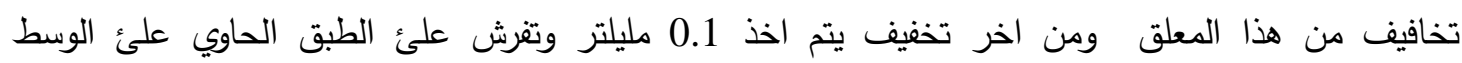

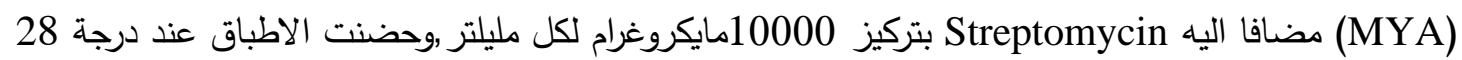
مُ ويتم مراقبة النمو بعد 24 ساعة من التحضين لحين الحصول علئ نمو جيد بعد 3-7 ايام.

6- تنقية وتثخيص الخمائر جرى تتقية المستعرات الخميرية على الوسط MYA ثم حفظت كل عينة نقية على سطح مائل في الثلاجة لاجراء الدراسات اللاحقة عليها. و جرى تشخيص العينات الخميرية مظهرياً ومجهرياً بالاعتماد على المفتاح التصنيفي لكل من pitt و Hocking [15] وملاحظة النموعلى الاوساط التفريقية السابقة الذكر كما وجرى باليات تميز الخمائر البازيدية عن الخمائر الكيسية باستخدام الكاشف الكيميائي DBB [16] ·و كذلك استخدام جهاز الVitek 2 compact التشخيصي (مدينة اربيل).

7- اختبار القدرة التضادية للخميرة ضاند الفطريات المعزولة : واجريت هذه الدراسة بطريقتين

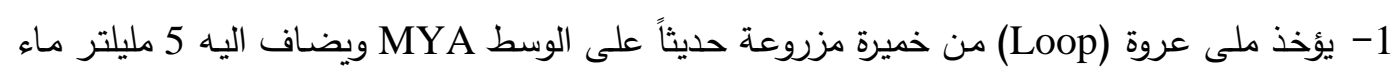

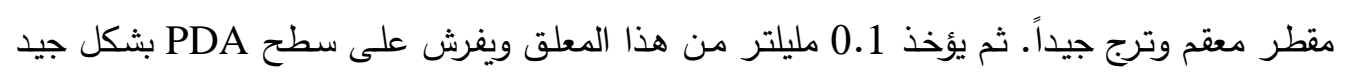

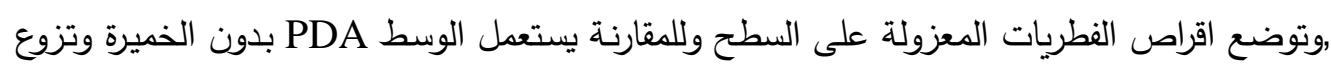
اقراص الفطر وتحضن في 28 مْ لحين امتلاء اطباق المقارنة:

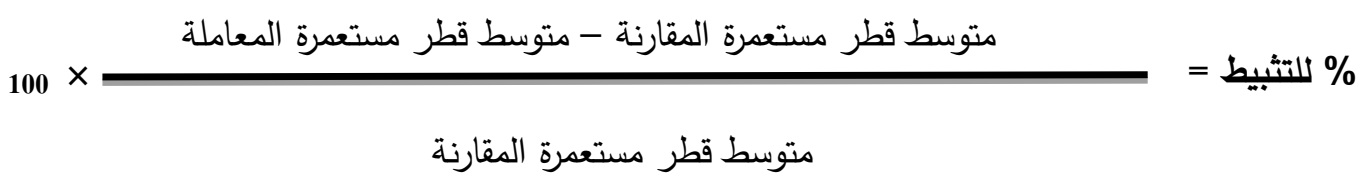

2- يتم رسم خط مستقيم بواسطة العرؤة Loop من مزرعة حديثة للخميرة بعمر يومين في احدى الاطراف او

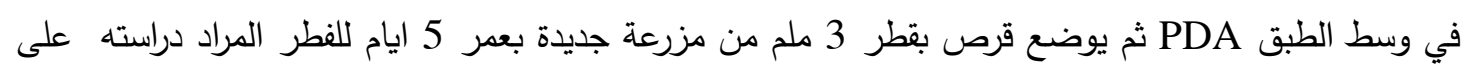

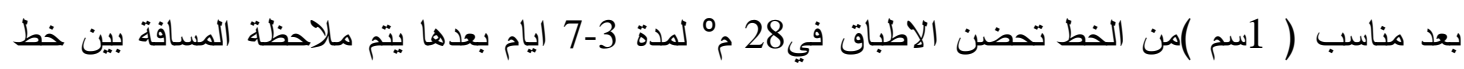

الخميرة وقرص الفطر (منطقة التثبي من اسن التبطن

\section{النتائج والمناقشة}

بعد العزل والتتقية جرى تثخيص الفطريات الى مستوى الجنس حصلنا على 8 اجناس

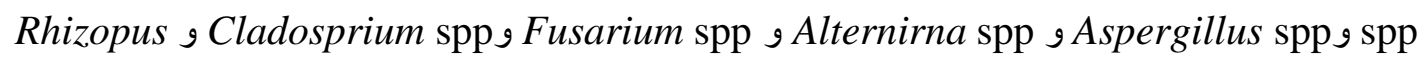

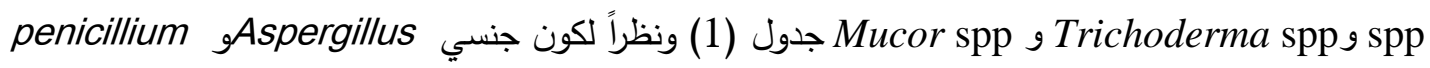
عزلت بتكرارات عالية فقد تم تشخيصها الى مستوى النوع 
جدول (1) يبين انواع الفطريات المعزولة ومصادر العزل:

\begin{tabular}{|c|c|c|}
\hline الفطريات المعزولة & الاسم العلمي للنبات & الاسم المحلية للنبات \\
\hline $\begin{array}{l}\text { Trichderma spp } \\
\text { Aspergillus niger } \\
\text { Aspergillus flavus } \\
\text { Aspergillus fumigitus } \\
\text { Aspergillus flavipes } \\
\text { Alternaria spp } \\
\text { Mucor spp }\end{array}$ & Citus sinensis & 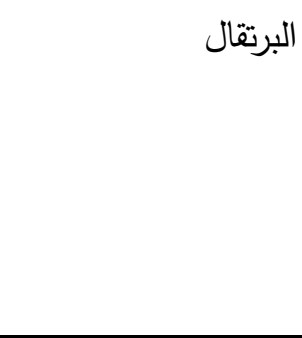 \\
\hline $\begin{array}{l}\text { Aspergillus flavus } \\
\text { Aspergillus niger } \\
\text { Penicillium digitatum } \\
\text { Penicillium italicum }\end{array}$ & Citrus limon & النوم الحامض \\
\hline $\begin{array}{l}\text { Aspergillus flavus } \\
\text { Aspergillus fumigitus }\end{array}$ & Citrus paradise & الكريب فروت \\
\hline $\begin{array}{l}\text { Alternira spp } \\
\text { Aspergllius niger } \\
\text { Rhizopus spp } \\
\text { Trichoderma } \mathrm{spp}\end{array}$ & Citrus reticulate & اليوسفي \\
\hline $\begin{array}{l}\text { Aspergillus flavus } \\
\text { Penicillium digitatum }\end{array}$ & Fortunella margarite & البرتقال الياباني \\
\hline $\begin{array}{l}\text { Penicillium digitatum } \\
\text { Aspergillus niger } \\
\text { Aspergillus fumigitus }\end{array}$ & Citrus aurantium & النارنج \\
\hline
\end{tabular}

كما وتم الحصول على 5 اجناس خميرية تعود الى Crptococcus و Candida وبينما لم يستطيع جهاز ال2 Vitek تشخيص Rhodotorula mucillaginosa و Geotrichum candidum إذْ جرى تشخيصها بالاعتماد على المفتاح التصنيفي[15]. وتم تشخيص الخمائر البازيدية والخمائر الكيسية بالاعتماد على الكاشف DBB وتبين أن 9 من العينات المعزولة كانت موجبة لهذا الكاشف اذا أعطت لون ارجواني واضح كما في الثكل (1) دليل على انها تعود إلى صنف الفطريات البازيدية، أما الخمائر الأخرى فتعود إلى صنف الفطريات الكيسية جدول (2): جدول (2) : الخمائر البازيدية والكيسية بأستخدام الكاشف DBB

\begin{tabular}{|c|c|}
\hline Diazonium fast blue & NY العي \\
\hline \multirow{2}{*}{+} & NY - 1 \\
& NY -4 \\
\hline- & NY-23 \\
\hline \multirow{2}{*}{-} & NY-2 \\
\hline+ & NY -3 \\
\hline \multirow{2}{*}{+} & NY-21 \\
\hline
\end{tabular}


رافعة قادر جرجيس و نسرين اسماعيل محمد

\begin{tabular}{|c|c|}
\hline Diazonium fast blue & العينة \\
\hline & NY-10 \\
\hline- & $\begin{array}{l}\text { NY }-6 \\
\text { NY-15 } \\
\text { NY-12 }\end{array}$ \\
\hline- & $\begin{array}{c}\text { NY -7 } \\
\text { NY-9 } \\
\text { NY-11 } \\
\end{array}$ \\
\hline+ & $\begin{array}{l}\text { NY }-8 \\
\text { NY-14 } \\
\text { NY-25 }\end{array}$ \\
\hline- & $\begin{array}{l}\text { NY }-16 \\
\text { NY-19 }\end{array}$ \\
\hline- & $\begin{array}{l}\text { NY }-17 \\
\text { NY }-20\end{array}$ \\
\hline- & $\begin{array}{l}\text { NY }-18 \\
\text { NY }-24 \\
\end{array}$ \\
\hline- & $N Y-26$ \\
\hline
\end{tabular}

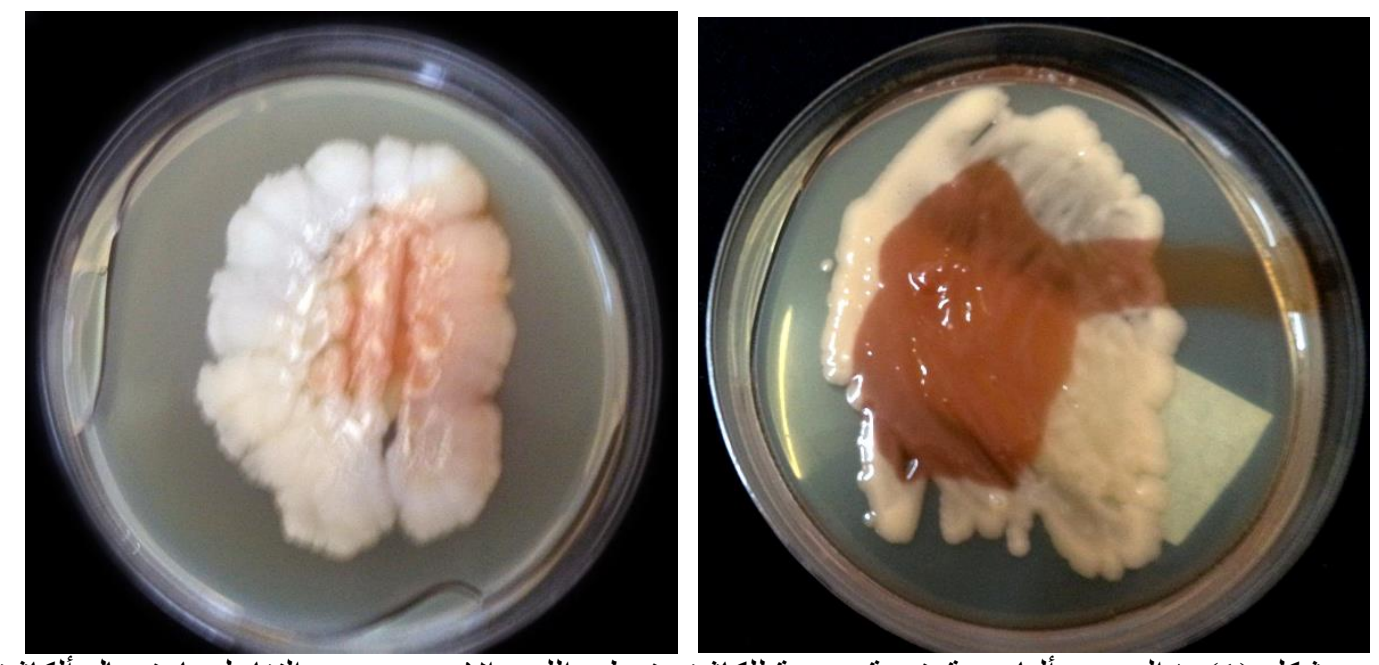

شكل (1) الخميرة ألبازيدية نتيجة موجبة للكاثف تعطي اللون الارجوني عند التفاعل باستعمال ألكاثف DBB

DBB الخميرة الكيسية نتيجة سالبة لاتعطي اي لون عند التفاعل مع الكاثفB

جدول (2) يبين نوع الخميرة واحتمالية الدقة لكل نوع لنظام Vitek2 Compact

\begin{tabular}{|c|c|c|}
\hline مصدر عزل الخميرة & الاحتمالية الدقة لنظام & نوع الخميرة \\
\hline يوسفي & 89 & Candida norvegensis \\
\hline يوسفي & $*$ & Rhodotorula mucillaginosa \\
\hline برتقال ياباني & 97 & Candida lambica \\
\hline 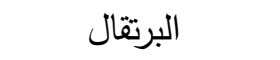 & 90 & Candida norvegensis \\
\hline
\end{tabular}




\begin{tabular}{|c|c|c|}
\hline مصدر عزل الخميرة & الاحتمالية الدقة لنظام & نوع الخميرة \\
\hline 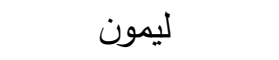 & 91 & Crptococcus laurentii \\
\hline 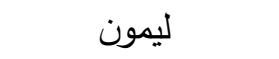 & 91 & Saccharomyces cerevisiae \\
\hline برتقال & 89 & Candida sphaerica \\
\hline يوسفي & $*$ & Unknown \\
\hline يوسفي & 91 & Candida sphaerica \\
\hline برتقال & 91 & Crptococcuslaurentii \\
\hline ل يوسفي & 98 & Candida sphaerica \\
\hline كريب فروت & 93 & Saccharomyces cerevisiae \\
\hline ل ليمون & 91 & Crptococcuslaurentii \\
\hline برتقال & * & unknown \\
\hline 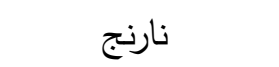 & 95 & Saccharomyces cerevisiae \\
\hline برتقال & 98 & Candida guilliermondii \\
\hline 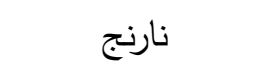 & 94 & Candida lusitaniae \\
\hline كريب فروت & 99 & Candida famata \\
\hline 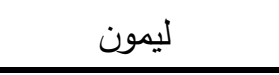 & 98 & Candida guilliermondii \\
\hline 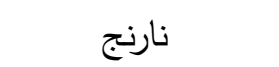 & 96 & Candida lusitaniae \\
\hline برتقال ياباني & 97 & Candida lambica \\
\hline ل ليمون & $*$ & Rhodotorula mucillaginosa \\
\hline 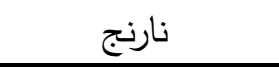 & 92 & Candida norvegensis \\
\hline كريب فروت & 99 & Candida famata \\
\hline برتقال ياباني & $*$ & unknown \\
\hline 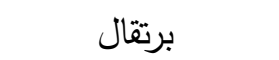 & $*$ & Geotrichum candidum \\
\hline
\end{tabular}

* لم يستطيع جهاز الـ Vitek 2 Compact من تشخيصها

توفر الحضيات وسط غذائي جيد لنمو الفطريات فالثمار الناضجة تكون غنية بالسكريات ودرجة حامضية

عالية وزيادة ليونة الفاكهة وارتفاع مستوى صبغات الكاروتين( Carotenoids) والمواد العطرية وانخفاض نسبة الكلوروفيل وهذه كلها عوامل تساعد على الإصـابة بالفطريات الممرضـ[117 كما ويشجع حدوث الإصابة وجود الجروح والخدش سواء أكانت مجهرية أم مرئية على الثمار التي تحدث خلال القطف والنقل مما يشجع العديد من الفطريات إلى اختراق نسيج الثمرة عن طريق جرح الجدار مما يسهل مهاجمة الفطريات لها وإحداث العفن

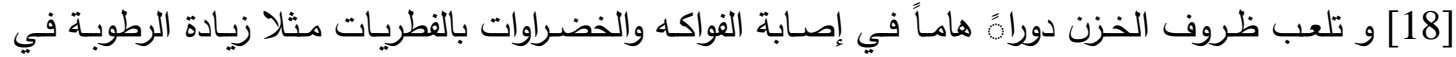

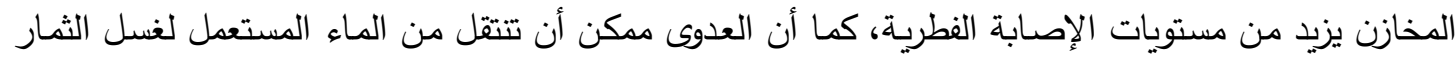


بعد الحصاد و الحاويات المستعملة لحفظ ونقل الفواكه والخضراوات [19] علاوة على امتلاك الفطريات لأقوى نظام أنزيمي يساعدها على تحليل جدران الخلايا النباتية من خلال إفراز أنزيمات محللة خارجية مثل بكتيزيز

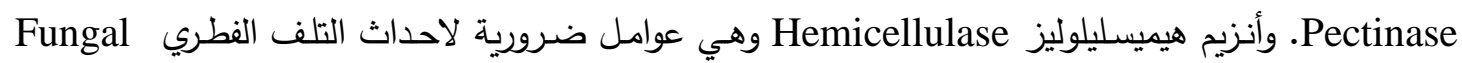
.[20] spoilage اختبار القدرة التضادية للخميرة Candida guiliermondii ضد الفطريات المعزولة

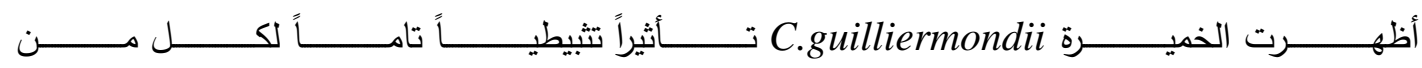
P. italicum A . niger

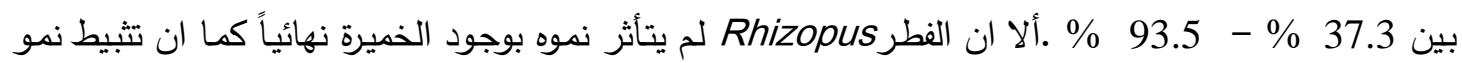

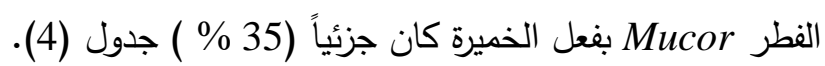
ان الخميرة C. guilliermondii تستخدم بشكل كبير في مجال المكافحة الحيويـة فقد استخدمت في مكافحة الفطر Penicillium digitatum قبل الحصاد وحققت نسب تثبيط لنمو الفطرات (21)، وهذا يتفق مع بـ نتائج دراستتا الحالية . والسبب يعود الى قدرة الخميرة على التتافس مع الفطريات الاخرى على المواد الغذائية كذلك انتاجها

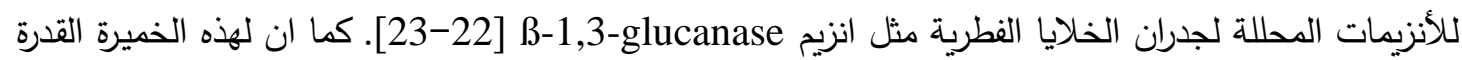

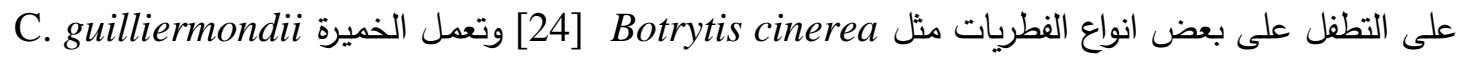
على تحفيز النباتات المضيفة لمقاومة الامراض الفطرية فقد تبين عند تلقيح نباتات الطماطة المثرة بسلالكة من

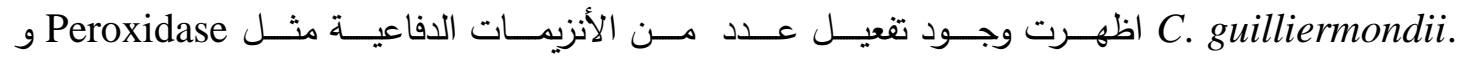
Polyphenoloxidase [25], وقد تعتمد الخميرة في استخدام اكثر من وسيلة لتثبط المسبب المرضي فهي في اكثر الاحيان تعتمد

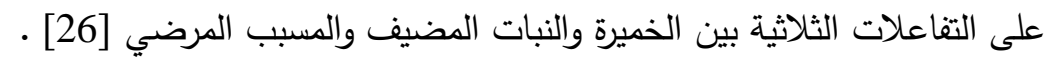
وفي دراسـة قام بها Zambrano واخرون 2727 حقق نسبة قتل للفطر Rhizopus stolonifer النامي على ثمار الطماطـة تصل الى 87\%. حيث استحوذت الخميرة على اكبر كمية من الغذاء ولم تسمح

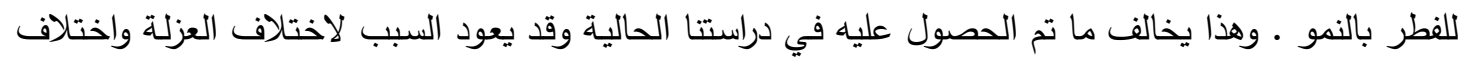
مكان العزل. وربما يكون استخدام اكثر من عامل حيوي واحد يخفض نسبة الاصابة وقد يعود السبب الى التأثير التآزري فيما بينها في تحفيز المقاومة الجهازية للنبات وتحفيز نمو النبات والسيطرة على مسببات امراض النبات وربما يعود السبب الى التداخل في اليات عملها مما ادى الى زيادة تحفيز نمو النبات والسيطرة على امراض النبات [28]

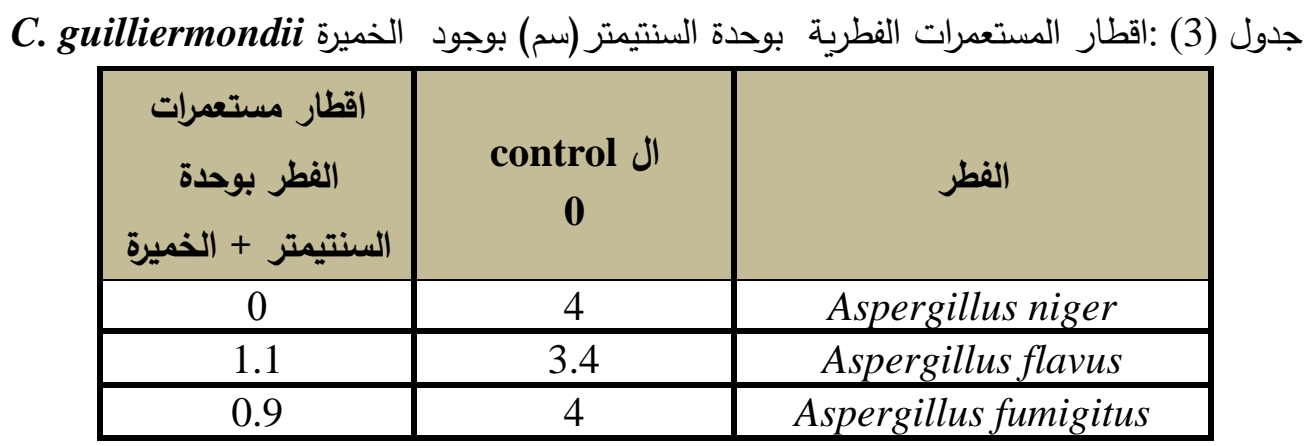




\begin{tabular}{|c|c|c|}
\hline الفطار مستعمرات الفطر بوحدة & $\begin{array}{c}\text { control ال } \\
0\end{array}$ & الفطر المر \\
\hline 0.45 & 2 & Aspergillus flavipes \\
\hline 0.2 & 3.1 & Penicillium digitatum \\
\hline 0 & 1.5 & Penicillium italicum \\
\hline 0.4 & 4.5 & Alternaria spp \\
\hline 0.7 & 3.1 & Cladosporium spp \\
\hline 0.6 & 9 & Trichoderma spp \\
\hline 9 & 9 & Rhizopus spp \\
\hline 2 & 9 & Fusarium spp \\
\hline 5.8 & 9 & Mucor spp \\
\hline
\end{tabular}

جدول (4) النسبة المئوية لتثبط نمو الاعفان بعل الخميرة

C. guilliermondii

\begin{tabular}{|c|c|}
\hline \% للتبيط للخميرة & الفطر الفر \\
\hline$\% 100$ & Aspergillus niger \\
\hline$\% 67.3$ & Aspergillus flavus \\
\hline$\% 77.2$ & Aspergillus fumigitus \\
\hline$\% 77.3$ & Aspergillus flavipes \\
\hline$\% 93.5$ & Penicillium digitatum \\
\hline$\% 100$ & Penicillium italicum \\
\hline$\% 90$ & Alternaria spp \\
\hline$\% 77.3$ & Cladosporium spp \\
\hline$\% 93.5$ & Trichoderma spp \\
\hline$\% 0$ & Rhizopus spp \\
\hline$\% 77.2$ & Fusarium spp \\
\hline$\% 35$ & Mucorspp \\
\hline
\end{tabular}


1. الاسود, ماجد بشير؛ عمر ,فوزي عبد العزيز؛ سولاقا ,امجد بويا ," مبادئ الصناعات الغذائية" ,دار الكتب

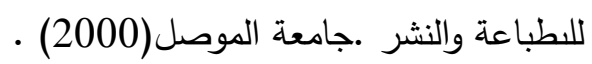

2. Wisniewski, M.E. ; Biles, C.and Droby, S. , US Department of Agriculture, Washington, DC, pp. 167-183. ( 1990).

3. Arekemase ,M.O. and Oyeyiola, G.P. , Centrepoint (Science Edition)14:138-149 (2007).

4. Akinmusire, O.O, Adv.Enviro.Biol., 5: 157-161 (2011).

5. Li, Z.; Alli, I. and, Kermash, S ., J . Fd.Sci . 54 : 674-678. ( 1989).

6. Winniczuk, P. and Parish , E ., ( Abst ) Fd .Microbiol ., 14 : 373 - 381 ( 1997 ).

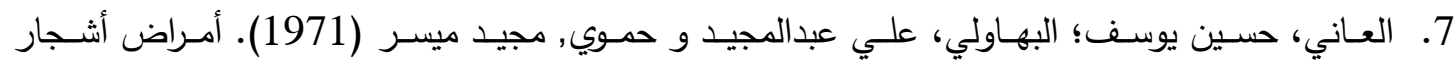

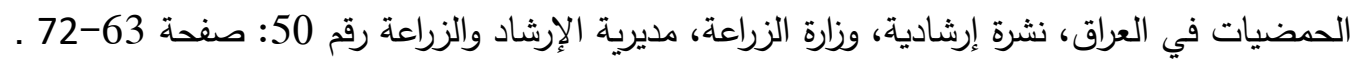

8. الكعبي، حوراء نعمة حسين. ، رسالة ماجستير، الكلية التقنية , المسيب, العراق, (2013).

9. Kohmoto , K . ; Scheffer , R . P . and Whiteside, J.O., J.of phytopath ., 69 : 667671. ( 1979 ).

10. Roland , J.O.; Beuchat, L.R. ; Worthington , R.E. and.Hitchcock, H.L, . J.Fd.prot .47: 237-241 .

11. Jay,J.M. Modern food micrology .3 rded . Nostrand Reinhold company C.B.S. Publishing New York 644pp. (1984) .

12. King, A.D. and Tarok, T. , Elsirier New York 4: 39-46 (1996).

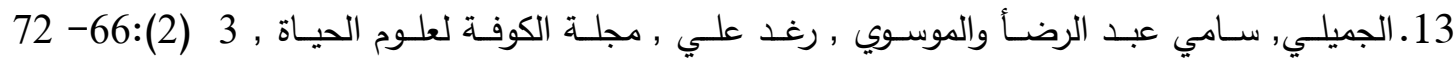

14.تيمور , سولاف حامد؛ عبدالرضا, ولاء عباس؛ مسلم,طيف مظهر وكريم, مالك علي ، مجلة القادية للعلوم

$$
\text { الصرفة, } 15 \text { (4): 1-6), (2010). }
$$

15. Pitt, J. I. and Hocking, A. D., Fungi and food spoilage springer. Business, New York. USA, (2009).

16. Ghosh, S.K. ; Santra , T. and Chakravarty , AA ,Agri. andBiol J. of north America , 4(1):33-40, (2013).

17. Wills, R. B. H. ; McGlasson, W. B. ; Graham, D. ; Lee, T. H. and Hall, E. G., Postharvest: an introduction to the physiology and handling of fruit and veg etables, $3^{\text {rd }}$. edition. University of new. South wales press, Sydney(1989).

18. وبيستر، جون (1980). مدخل إلى الفطريـات، ترجمـة الدكتور إبر اهيم عزيز خالـد السـهيلي، مطبعـة جامعة بغداد، 638 صفحة

19. Coates, L.; cooke, A. ; Persley, D. ; Beattie, B. ; Wade, N. and Ridgeway, R. volume 2: tropical fruit. DPI, Queensland, (1995).

20. Miedes, E. and Lorences, E. P.., Journal of Agricultural and food chemistry, 52: 7957- 7963. (2004).

21. Droby, S.; Chalutz, E.; Wilson, C. L. and Wisniewski, M. Can. J. microbial, 35: 794- 800, (1989). 
22. Chanchaichaovirat, A. ; Ruenwongsa, P. and Panijpan, B., Biolo. controlJ., 42: 326-335 (2007).

23.Zhang , D.P. ;Spadaro ,D. ;Valente, S. ; Garibaldi, A. and Gullino, .L,Biol.Control,59:284-293,(2011).

24. Wisniewski, M.E. ; Biles, C.and Droby, S., US Department of Agriculture, Washington, DC, pp. 167-183, ( 1990) .

25.Zhao, Y. ; Tu, K. ; Shao, X.F. ;Jing ,W. and Su, Z.P., Postharvest Biol.Technol,49:113-120, (2008).

26. Liu, F.J. ; Tu, K. ; Shao ,X.F. ; Zhao ,Y. ;Tu ,S.C. ; Su, J. ;Hou ,Y.P.and Zou ,X.R., Postharvest Biol Technol 58:65-71, (2010).

27.Zambrano, C. C. ; Duran, G. M. and Castaüeda L. G., Univ. Sci:, 19(1):51-260, (2014).

28. Thilagavathi, R. ; Saravanakumar ,D. ; Ragupathi, N. and Samiyappan, R., Phytopathology Mediterranean, 46 157-167. (2007) . 